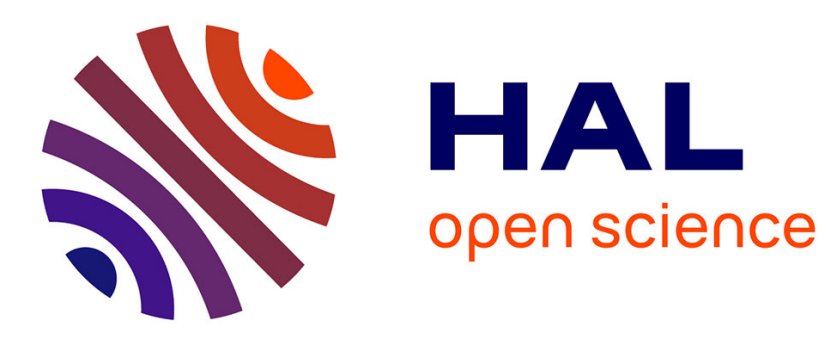

\title{
Synergy between the crack pattern and substrate elasticity in colloidal deposits
}

Hisay Lama, Tonmoy Gogoi, Madivala Basavaraj, Ludovic Pauchard, Dillip Satapathy

\section{- To cite this version:}

Hisay Lama, Tonmoy Gogoi, Madivala Basavaraj, Ludovic Pauchard, Dillip Satapathy. Synergy between the crack pattern and substrate elasticity in colloidal deposits. Physical Review E , 2021, 103 (3), pp.032602. 10.1103/PhysRevE.103.032602 . hal-03294506

\section{HAL Id: hal-03294506 https://hal.science/hal-03294506}

Submitted on 8 Nov 2021

HAL is a multi-disciplinary open access archive for the deposit and dissemination of scientific research documents, whether they are published or not. The documents may come from teaching and research institutions in France or abroad, or from public or private research centers.
L'archive ouverte pluridisciplinaire HAL, est destinée au dépôt et à la diffusion de documents scientifiques de niveau recherche, publiés ou non, émanant des établissements d'enseignement et de recherche français ou étrangers, des laboratoires publics ou privés. 


\title{
Synergy between the crack pattern and substrate elasticity in colloidal deposits
}

\author{
Hisay Lama, ${ }^{1,2,3}$ Tonmoy Gogoi, ${ }^{1}$ Madivala G. Basavaraj, ${ }^{2}$ Ludovic Pauchard, ${ }^{4}$ and Dillip K Satapathy ${ }^{1, *}$ \\ ${ }^{1}$ Soft Materials Laboratory, Department of Physics, IIT Madras, Chennai-600036, India \\ ${ }^{2}$ Department of Chemical Engineering, IIT Madras, Chennai, India \\ ${ }^{3}$ IBS Center for Soft and Living Matter, UNIST, Ulsan-44919, South Korea \\ ${ }^{4}$ Laboratoire FAST, Univ. Paris-Sud, CNRS, Université Paris-Saclay, F-91405, Orsay, France
}

(Dated: February 19, 2021)

\begin{abstract}
Desiccation cracks in colloidal deposits occur to release the excess strain energy arising from the competition between the drying induced shrinkage of the deposit and its adhesion to the substrate. Here, we report remarkably different morphology of desiccation cracks in the dried patterns formed by the evaporation of sessile drops containing colloids on elastomer (soft) or glass (stiff) substrates. The dramatic change in the crack pattern, i.e., from radial cracks on stiff substrates to circular cracks on soft substrates, is shown to arise solely due to the variation in elasticity of the underlying substrates. Our experiments and calculations reveal an intricate correlation between the desiccation crack patterns and the substrate's elasticity. The mismatch in modulus of elasticity between the substrate and that of the particulate deposit significantly alters the energy release rate during the nucleation and propagation of cracks. The stark variation in crack morphology is attributed to the tensile or compressive nature of the drying-induced in-plane stresses.
\end{abstract}

\section{INTRODUCTION}

Cracks occur naturally in drying solids and often attractive patterns emerge from their spontaneous selforganization. For example, periodic cracks observed in layered limestones $[1,2]$, roughly hexagonal cracks with Y-junctions in dried mud [3, 4], the cracking of cooling lava into regular prismatic columns $[5,6]$ are beautiful crack patterns found in nature. On the other hand, a wide variety of crack patterns such as regular parallel cracks [7], wavy cracks [8], and spiral cracks [9] are observed in the laboratory by drying colloidal dispersions. Interestingly, the length scales of cracks occurring in nature, albeit being very different than that formed in the laboratory experiments, the crack spacing in both the cases comprehend by a simple scaling theory [6]. Thus, the study of desiccation crack patterns in drying colloids and their kinetics can provide powerful insight into the underlying physics over a wide range of length and time scales [10]. When a drop of colloidal dispersion dries on a solid surface, the evaporation-driven consolidation of colloids tends to shrink the particulate deposit, whereas its adhesion to the substrate resists this shrinkage. The interplay between the shrinkage and adhesion resistance leads to the accumulation of strain energy within the particulate deposit. When the accumulated internal stress equals or exceeds the magnitude of the critical stress $\sigma_{c}$, the crack nucleates $[3,7,11-14]$. The critical stress, $\sigma_{c}$, and the energy release rate, $G$, associated with cracking of the particulate deposit can be estimated by invoking Griffith's criterion [15]. The energy release rate, i.e., the energy available per unit area for cracking, depends on both the elastic modulus of the substrate $\left(\mathrm{E}_{\mathrm{s}}\right)$ and the particulate film $\left(\mathrm{E}_{\mathrm{f}}\right)[16]$. There exist a plethora of studies that investigate the patterns of desiccation cracks and

\footnotetext{
*dks@iitm.ac.in
}

its correlation with the various aspects such as physicochemical condition [17], size of particles [12] and thickness of the particulate deposit $[7,10]$. But, the role of the substrate's elasticity on drying induced crack patterns is hardly explored [18], and still remains elusive.

In this article, we report a striking difference in the desiccation crack pattern, when sessile drops containing colloids are dried on elastomer (soft) substrates and on glass (stiff) substrate. We show that the mismatch in the modulus of elasticity of the substrate $\left(\mathrm{E}_{\mathrm{s}}\right)$ and the particulate deposit $\left(\mathrm{E}_{\mathrm{f}}\right)$ strongly influences the nucleation and the morphology of desiccation cracks. Radial cracks, i.e., cracks primarily oriented along the radial direction, form for drops dried on stiff substrates, whereas circular cracks (cracks in the form of concentric circles) appear for drops dried on soft substrates. The dramatic change in crack morphology from radial-to-circular is explained by taking into account the relative distribution of the drying induced in-plane stresses in the colloidal deposits on stiff and soft substrates respectively. It is worth mentioning that the coexistence of radial and circular cracks in dried deposits has been reported in the past [19]. The formation of circular and radial crack patterns are related to the local microstructure of the particulate deposit. However, in the present study, we show that a transition from radial to circular cracks can be achieved solely by varying the elastic modulus of the substrate.

\section{MATERIALS AND METHODS}

For the desiccation experiments, we use aqueous dispersion of spherical silica colloids (Ludox TM-50) with a diameter $d=32 \pm 2 \mathrm{~nm}$ and a zeta potential $(\zeta)$ of $-39 \mathrm{mV}$. The particles in the disperison are charge stabilized with the stock dispersion consisting $50 \%$ of particles by weight. A dispersion of desired concentration is obtained by suitably diluting the stock dispersion with 


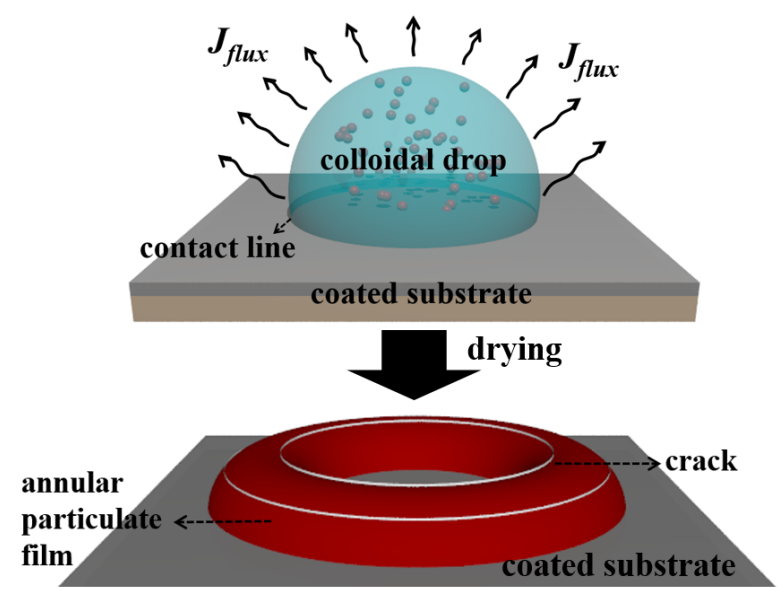

FIG. 1. (Top) Schematic displaying an aqueous sessile drop placed on a solid substrate. The non-uniform evaporative flux $J_{\text {flux }}$, the solid substrate, and the three-phase contact line are marked. (Bottom) Schematic of the annular particulate deposit with circular cracks formed after complete evaporation of the solvent.

milli-Q water of resistivity $\rho \sim 18.2 \mathrm{M} \Omega$-cm. Prior to the drying experiments, the colloidal dispersion is always sonicated in an ultra-sonicator in order to obtain a well mixed homogeneous dispersion. The substrates used in the experiments are (i) glass coated with $219 \pm 4 \mu \mathrm{m}$ thick poly-di-methyl siloxane (PDMS), henceforth referred as 'soft substrate' or (ii) glass coated with silane (stiff substrate).We present below - (a) the fabrication process of these substrates, (b) details on desiccation experiments and (c) description on the measurement of Young's modulus:

Soft substrates: The soft substrates are fabricated by coating poly-di-methylsiloxane (PDMS, Sylgard 184, Dow Corning, USA) elastomer on a microscopy glass slide. Typically, two components, the PDMS (base) and the cross-linker (curing agent) are hand mixed in 10:1 mass ratio. The blend being highly viscous traps air during mixing resulting in unwanted air bubbles in the sample. These air bubbles are removed by degassing the mixture using a vacuum desiccator. Once the bubbles are completely removed, the mixture is used for the preparation of PDMS film. The mixture is poured on a precleaned dry glass slide of $2.5 \mathrm{~cm} \times 2.5 \mathrm{~cm}$ dimension and spin-coated (POLOS ${ }^{\mathrm{TM}}$ - spin coater) at a speed of 1000 $\mathrm{rpm}$ with an acceleration of $100 \mathrm{rpm} / \mathrm{s}$ for about $40 \mathrm{~s}$. Finally, the coated films are cured for 15 minutes in a hot air oven maintained at temperature of $150^{\circ} \mathrm{C}$. The contact angle of water on these PDMS coated glass substrate is measured to be $\theta_{c} \approx 117^{\circ}$. The thickness of the PDMS films deposited on glass substrates is measured to be $100 \pm 10 \mu \mathrm{m}$, by using a micrometre screw gauge (Mitutoyo, Digimatic Micrometer).

Stiff substrates: The stiff substrates are prepared by coating microscopy glass slides with the alcosol that com-
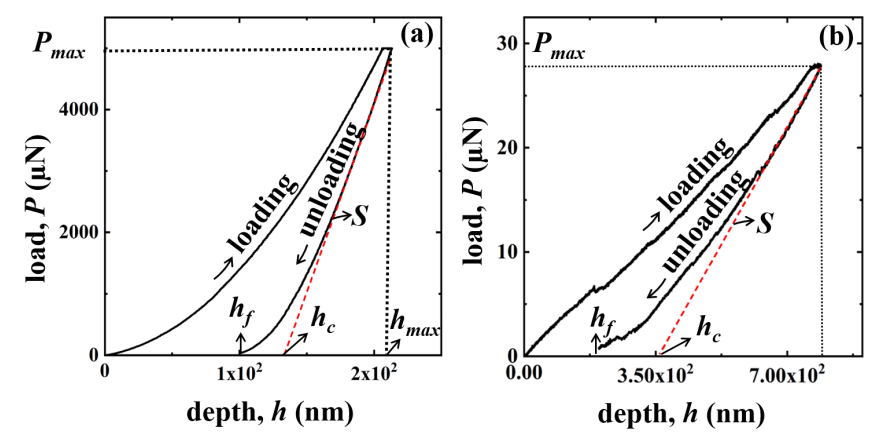

FIG. 2. Load vs displacement plots measured for (a) glass substrate and (b) glass substrate coated with a PDMS layer. The slope $S$ of the unloading part of the load-displacement curve, maximum penetration depth $h_{\max }$, contact depth $h_{c}$, residual depth after unloading $h_{f}$ and the maximum load $P_{\max }$ are indicated. The red dotted line, guide to the eye, shows the tangent to the unloading part of the curve drawn at $\left(P_{\max }, h_{\max }\right)$. The estimated values of $E_{s}$ for glass and glass coated with PDMS elastomer are around to be $70 \mathrm{GPa}$ and $6 \mathrm{MPa}$ respectively.

prises methyltriethoxysilane, methanol and ammonium hydroxide $\left(\mathrm{NH}_{4} \mathrm{OH}\right)$ in 1:35.22:2.01 molar ratio. The methodology for preparing the alcosol solution comprises of - diluting methytriethoxysilane (MTES) in methanol $\left(\mathrm{CH}_{3} \mathrm{OH}\right)$ and then adding specific amount of $\mathrm{NH}_{4} \mathrm{OH}$ to the mixture followed by stirring of the mixture for an hour with the help of a magnetic stirrer. Then the mixture is left undisturbed at room temperature for about 12 hours. Thereafter, the solution (alcosol) is spin-coated on the glass substrate at a spin speed of $1000 \mathrm{rpm}$ at an acceleration of $100 \mathrm{rpm} / \mathrm{s}$ for $40 \mathrm{~s}$. The alcosol coated slides are annealed at a temperature of $60^{\circ} \mathrm{C}$ for 5 minutes in a hot air oven. Next, a second alcosol layer is spin coated on the glass slides using the same procedure and further annealed at $150^{\circ} \mathrm{C}$ for about two hours. The contact angle of water on these silane coated glass substrates is measured to be $\theta_{c} \approx 111 \pm 2^{\circ}$, nearly same as that on the glass substrate coated with PDMS [20].

Desiccation experiment: The desiccation experiments are performed in sessile drop configuration with the droplet volume $V$ varying from $1 \mu \mathrm{L}-6 \mu \mathrm{L}$ and particle concentration, $\phi \sim 3 \mathrm{wt} \%$, a schematic of which is in Fig. 1. The evaporation experiments are performed in an environment at temperature, $\mathrm{T}_{\text {env }} \approx 25 \pm 2^{\circ} \mathrm{C}$ and relative humidity, $\mathrm{RH} \approx 40 \pm 5 \%$. The drying of colloids in the sessile configuration yields a narrow annular deposit of colloidal particles, commonly known as the coffee-ring [21]. The ring-like deposit of colloids arises due to the radial outward capillary flow caused by the non-uniform evaporative flux across the drop surface $[21,22]$. The deposit of colloids transforms into a gel-like phase prior to the nucleation of cracks. The process of nucleation and propagation of cracks in the dried ring-like deposits of colloids are monitored using an optical microscope (Leica, Model: DMI3000B, 


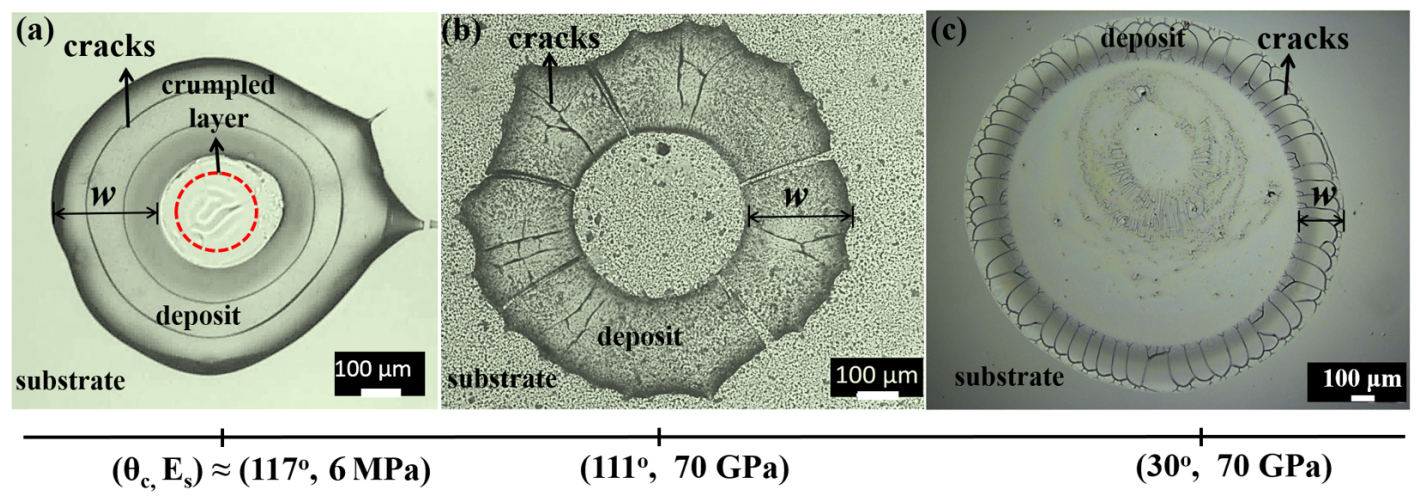

FIG. 3. Optical microscopy images of the annular colloidal deposits exhibiting (a) circular cracks on soft substrate, (b) radial cracks on silane coated stiff substrate and (c) radial cracks cleaned glass substrate. The crack patterns are consistently reproducible for at least ten different trial experiments. The contact angle, $\theta_{c}$ subtended by a drop of water and the modulus of elasticity $\mathrm{E}_{\mathrm{s}}$ of various substrates are marked. The width $w$, of the cracks in particulate deposits and the crumpled region of soft substrate are marked.

Germany) operated in the bright field mode. The thickness of dried particulate deposit and the deformed region on the soft substrates are measured using an optical surface profile-meter (Bruker, Germany). Note that drying in sessile drop configuration is considered in this work as the cracks formed for a given set of control parameters (e.g., contact angle, droplet volume, $\mathrm{pH}$, and other evaporation conditions) are distinct and highly reproducible [23, 24]. Moreover, the drop drying in this configuration is relevant to various applications, ranging from medical diagnostics, forensics, food technology, and functional coating of surfaces [25].

Indentation measurement: The Young's modulus of the glass coated PDMS elastomer (soft) and the glass (stiff) substrates are measured using Hysitron TI Premier nanomechanical tester fitted with diamond indenter tip. The load versus displacement curves are recorded at six different locations chosen randomly on the surface of these substrates. In the nano-indentation experiments, the specimen is subjected to a vertical load from 0 up to a maximum load $\left(P_{\max }\right)$ at a rate of $5 \mu \mathrm{N} / \mathrm{s}$. Subsequently, the load is withdrawn at a rate of $10 \mu \mathrm{N} / \mathrm{s}$ after a holding period of $10 \mathrm{~s}$. The Young's modulus is estimated form the load-displacement ( $P$ vs $h$ ) plots by using Oliver Pharr model [26] from the slope of the tangents shown in Fig. 2(a) and Fig. 2(b). The reduced modulus $E_{r}$ is estimated from [27]:

$$
E_{r}=\frac{\sqrt{\pi}}{2 \sqrt{A} \beta}\left(\frac{d P}{d h}\right)_{h=h_{\max }}
$$

where, the coefficient $\beta$ is a constant, $A$ is the contact area and $d P / d h$ is the slope of the unloading curve at $h=h_{\max }$ (maximum penetration depth). For Berkovich indenter, $\beta=1.034$ and $A=24.5 h_{c}^{2}$ where $h_{c}$ is the contact depth estimated using the Oliver Pharr model $[26,27]$. The Young's modulus, $E_{s}$ is calculated using the expression [27],

$$
E_{s}=\frac{1-\nu_{s}^{2}}{\frac{1}{E_{r}}-\frac{1-\nu_{i}^{2}}{E_{i}}}
$$

where, $\nu_{s}$ and $\nu_{i}$ are the Poisson's ratios of the specimen and the indenter tip respectively, $E_{i}$ is the Young's modulus of an indenter tip. Note that for a given measurement $\nu_{s}, \nu_{i}(=0.07)$ and $E_{i}(=1140 \mathrm{GPa})$ are all known physical quantities. The estimated magnitudes of Young modulus for the glass substrate coated with PDMS and the glass substrate is found to be around 6 $\mathrm{MPa}$ and $70 \mathrm{GPa}$ respectively.

\section{RESULTS}

\section{A. Cracks Pattern}

We first discuss the variation in the crack patterns observed for sessile drops dried on the soft and stiff substrates, shown in Fig. 3. In the annular particulate deposits on the soft substrate, circular cracks are formed, as shown in Fig. 3(a). In contrast, colloidal deposits on the stiff substrates always exhibit radial cracks, as depicted in Fig. 3(b). For clarity, a sessile drop of colloids is dried on a pristine glass substrate i.e., without any coating, and the resulting radial crack pattern is shown in Fig. 3(c). In both types of stiff substrates, silane coated glass substrates having a matching contact angle with the soft substrate, $\theta_{c}=111^{\circ}$ and the cleaned glass with $\theta_{c}=30^{\circ}$, the radial cracks are observed. The physical parameters that characterize the crack pattern such as the width of the deposit $(w)$, the mean crack spacing $(\lambda)$, radius $R$ i.e., the distance from the centre of the drop to the exterior edge of the dried deposit and $H_{f}$, the average thickness of the annular particulate deposit, are listed in Table I. The width and average thickness of 
TABLE I. List of parameters estimated from the optical microscopy images in Fig. 3. The initial particle concentration, $\phi \sim 3 \mathrm{wt} . \%$ and volume of the sessile drop is $2 \mu \mathrm{l}$.

\begin{tabular}{l||c|c|c}
\hline & PDMS coated & silane coated & pristine glass \\
variable & $\mathrm{E}_{\mathrm{s}} \approx 1 \mathrm{MPa}$ & $\mathrm{E}_{\mathrm{s}} \approx 70 \mathrm{GPa}$ & $\mathrm{E}_{\mathrm{s}} \approx 70 \mathrm{GPa}$ \\
\hline drying time, $t_{f}$ & $\sim 1200 \mathrm{~s}$ & $\sim 1200 \mathrm{~s}$ & $\sim 600 \mathrm{~s}$ \\
crack pattern & circular & radial & radial \\
radius, $R$ & $\sim 410 \mu \mathrm{m}$ & $\sim 465 \mu \mathrm{m}$ & $\sim 1196 \mu \mathrm{m}$ \\
width, $w$ & $\sim 210 \mu \mathrm{m}$ & $\sim 230 \mu \mathrm{m}$ & $\sim 200 \mu \mathrm{m}$ \\
thickness, $H_{f}$ & $\sim 62 \mu \mathrm{m}$ & $\sim 65 \mu \mathrm{m}$ & $\sim 15 \mu \mathrm{m}$ \\
crack spacing, $\lambda$ & $\sim 80 \mu \mathrm{m}$ & $\sim 350 \mu \mathrm{m}$ & $\sim 113 \mu \mathrm{m}$ \\
\hline
\end{tabular}

the dried annular particulate deposits formed on soft and stiff substrates are found to be nearly equal. However, the crack spacing $(\lambda)$ for the circular and radial cracks varies significantly i.e. $\lambda=80 \mu \mathrm{m}$ for soft substrates and about $\lambda=350 \mu \mathrm{m}$ for silane coated stiff substrate. As evident from Table I, the particulate deposit on the pristine stiff substrates exhibits radial cracks similar to those observed in the silane coated substrates, but with a different $\lambda$ and $H_{f}$. This directly implies that the radial or circular morphology of cracks is independent of the contact angle $\theta_{c}$ but is dependent on the elastic modulus of the substrate. The formation of circular cracks in the particle deposits on the soft substrates are verified for a wide range of initial volumes varying from $1 \mu \mathrm{L}$ up to 6 $\mu \mathrm{L}$ and for the thickness of PDMS from a few hundreds of micrometer down to a few micrometers. The circular morphology of cracks in these particulate deposits are found to persist with thickness as low as $13 \mu \mathrm{m}$. Interestingly, the circular cracks in the colloidal deposits on the soft substrate is accompanied by a crumpled region observed at the central region of the drop and is highlighted by a dotted circle in Fig. 3(a). We emphasize that the crumpling of the substrate always precedes the formation of circular cracks. The crumpling of the substrate and its three-dimensional surface profile depicting the topography of the crumpled region at the center of the dried deposit is shown in Fig. 4(a) and Fig. 4(b) respectively. The crumpling highlights the shrinkage of the drying colloidal deposit and its effect on the underlying soft substrates. Further, the maximum out of plane deflection of the substrate due to crumpling is measured to be around $4 \pm 1 \mu \mathrm{m}$, as shown in Fig. 4(b). Next the detailed description on propagation of cracks on these substrates (soft or stiff) are presented.

\section{B. Crack Propagation}

Propagation of crack on soft substrates The nucleation and propagation of cracks in the annular particulate deposit on a soft substrate are depicted in the bright field optical microscopy images shown in Fig. 5(a) - Fig. 5(h). The time $t_{c}$ marked in the Fig. 5 indicates the time at which the colloidal dispersion solidifies and

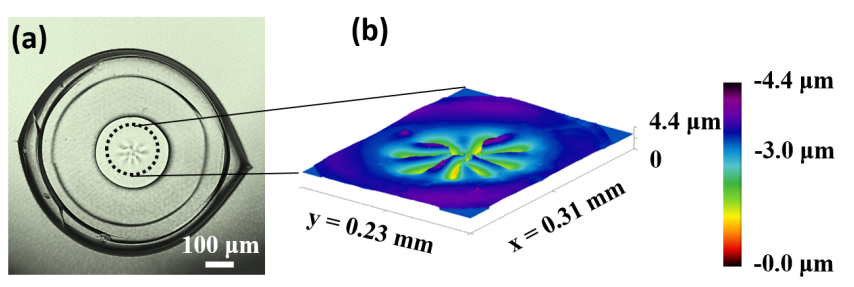

FIG. 4. (a) The optical microscopy image of the dried annular particulate deposit on a soft substrate with an accompanying crumpled layer (highlighted inside the dotted circle). (b) The corresponding height profile of the crumpled layer measured with an optical surface profile meter. The color bar indicates the height along the out-of-plane direction.

transform into a gel-like (semi-solid) annular deposit of width $w \approx 200 \mu \mathrm{m}$ as shown in Fig. 5(a). Then at time $t_{c}+0.61 \mathrm{~s}$, the annular colloidal film shrinks radially inwards resulting in crumpling of the soft substrate beneath the particulate deposit. The crumpled region that forms on the soft PDMS substrate is highlighted by a dotted circle in Fig. 5(b). Later at time $t_{c}+0.94 \mathrm{~s}$, a crack nucleates from the point $A$ and propagates along the circumferential direction as shown in Fig. 5(c) and Fig. $5(\mathrm{~d})$. At later instant of time, $t_{c}+1.11 \mathrm{~s}$ and $t_{c}+1.17 \mathrm{~s}$ the crack propagates in the opposite direction starting from the nucleation point $A$, eventually resulting in the formation of a closed circumferential loop as shown in Fig. 5(e) and Fig. 5(f). At $t_{c}+1.51 \mathrm{~s}$, a second crack forms and propagates along the circumferential path as shown in Fig. 5(g). With the formation of the second circular crack, the cracking process completely ceases and the image of the ring-like particulate deposit with cracks is shown in Fig. 5(h). Interestingly, the process of nucleation and propagation of cracks occur in the last 3 s i.e., when the gel-like semi-solid deposits completely transform into a dried solid particulate deposit (the total desiccation time, $\left.t_{d} \sim 1200 \mathrm{~s}\right)$. The thickness of a dried particulate deposit is found to be around $50 \mu \mathrm{m}$.

Propagation of crack on stiff substrates The bright field optical microscopy images displaying the nucleation and propagation of cracks in the particulate deposits on a stiff substrate is shown in Fig. 6. As mentioned earlier, at time $t_{c}$, the particulate deposit is in a gel-like semi-solid state with the width $w \approx 200 \mu \mathrm{m}$ as shown in Fig. 6(a). At time $t_{c}+0.27 \mathrm{~s}$, the crack nucleates from the point $A^{\prime}$ and propagates in the radial direction as shown in Fig. 6(b). As time progresses, between $t_{c}+1.66 \mathrm{~s}$ and $t_{c}+3.28 \mathrm{~s}$, the nucleation of second, third, fourth and fifth crack occurs which further propagate along the radial direction as shown in Fig. 6(c) Fig. $6(\mathrm{f})$. Finally at $t_{c}+4.95 \mathrm{~s}$ and $t_{c}+6.68 \mathrm{~s}$ these cracks widen followed by the the nucleation of a few narrower radial cracks. Similar to soft substrates, the process of nucleation and propagation of crack occurs at the tail end of drying process within the last $6 \mathrm{~s}$ of drying. The total drying time is about $t_{d} \sim 1200 \mathrm{~s}$. Note that for both the cases, i.e., for the colloids deposited on the soft and 


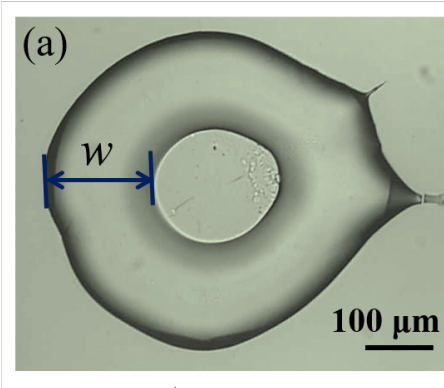

$t_{c}$
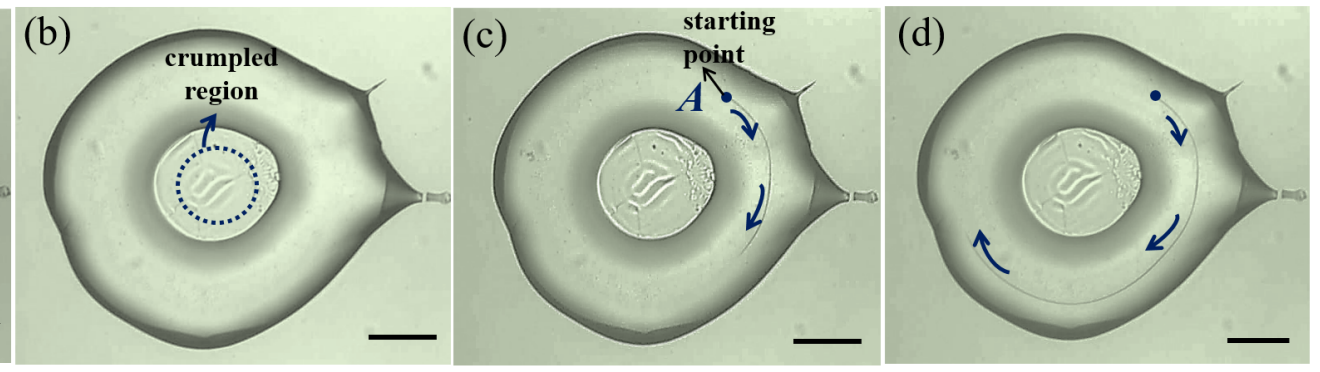

$\mathrm{t}_{\mathrm{c}}+0.61 \mathrm{~s}$

$\mathrm{t}_{\mathrm{c}}+0.94 \mathrm{~s}$

$\mathrm{t}_{\mathrm{c}}+1.0 \mathrm{~s}$

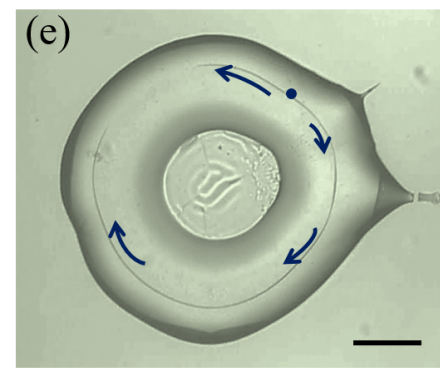

$\mathrm{t}_{\mathrm{c}}+1.11 \mathrm{~s}$

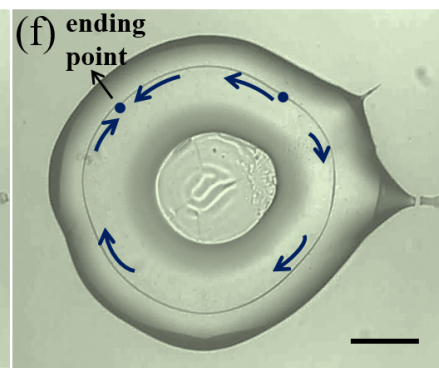

$\mathrm{t}_{\mathrm{c}}+1.17 \mathrm{~s}$

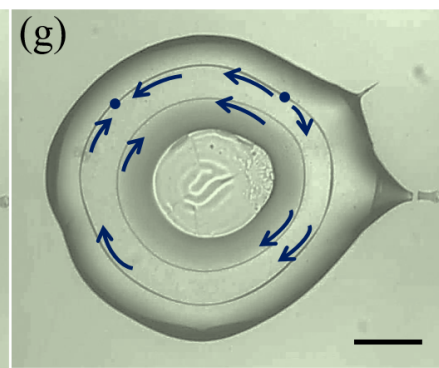

$t_{c}+1.51 \mathrm{~s}$

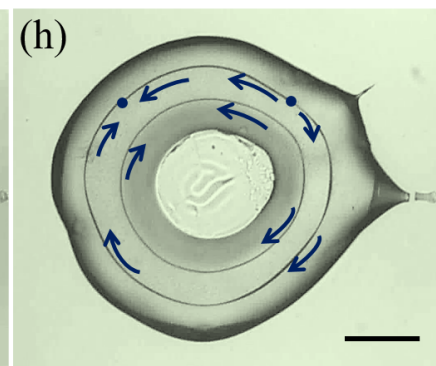

$\mathrm{t}_{\mathrm{c}}+3.5 \mathrm{~s}$

FIG. 5. (a) - (h) Snapshots recorded during the last stages of the drying process. The snapshots are extracted from the video-microscopy movie (see Supplementary Movie S1) recorded during the drying of a colloidal drop deposited on the soft substrate $\left(\mathrm{E}_{\mathrm{s}} \sim 6 \mathrm{MPa}\right)$. The morphological evolution of the crack patterns formed in the particulate deposit at $t_{c}$, the time at which colloidal dispersion transforms into a gel-like film and at various instant thereafter are shown. The width $w$ of the deposit, and the crack nucleating point $A$ are marked. The direction of propagation of cracks is highlighted with arrows. The crumpling of the soft substrate is also marked by a dotted circle at the central region of the ring-like deposit.

stiff substrates, the propagation of cracks persists as the deposit transforms from the gel-like semi-solid state into a completely dry solid particulate deposit. Besides, as evident from the video microscopy images showing the morphological evolution of cracks, we observe that the cracks in the particulate deposits always nucleate at the tail end of drying (last 4-6 s) irrespective of soft or stiff substrates (shown in supplementary video Movie S1 and Movie S2 [28]).

\section{DISCUSSION}

Further insight into the stark difference in the crack patterns in the deposits formed on soft and stiff substrates, has been obtained by estimating the energy release rate ' $G$ ', which is the energy available for the formation of new unit surface area by the crack. The $G$ is estimated using the Xia-Hutchinson model, developed for the propagation of parallel cracks arising from the mismatch between the modulus of elasticities of the brittle film and the substrate [16, 29]. This can be adopted for the particulate deposits reported here since the cracks in the particulate deposit nucleate at the tail end of drying i.e., when it is almost in a solid-like state (shown in Fig. 5 and Fig. 6) [12]. The stresses developed in the drying annular particle deposit can be approximated to be in-plane since the deposit is too thin to be influenced by any normal stress exerted by the air. The corresponding energy release rate $G$ associated with the propagation of cracks is given by [29]:

$$
G=\frac{l \sigma_{\circ}^{2}}{E_{f}} \tanh \left(\frac{\lambda}{2 l}\right)
$$

where, $\lambda$ is the crack spacing, $l=\frac{\pi}{2} g(\alpha, \beta) H_{f}$, is the characteristic crack length at which the stress generated in the particulate film (deposit) and the substrate decay, $\mathrm{E}_{\mathrm{f}}=1 \mathrm{GPa}[17]$ is the Young modulus of the particulate deposit, $\sigma_{\circ}\left(\approx-P_{\text {cap }} \sim-10^{7} \mathrm{~N} / \mathrm{m}^{2}\right)$ is the capillary pressure, $g(\alpha, \beta)$ is a function that depends on the Dundurs parameters $\alpha$ and $\beta$ (provided in the Appendix) $[16,28,30]$. The $\alpha, \beta$ are the dimensionless numbers that depend on $\mathrm{E}_{\mathrm{s}}, \mathrm{E}_{\mathrm{f}}$ and the Poisson's ratio of the particulate film $\left(\nu_{f}\right)$ and substrate $\left(\nu_{s}\right)$. The $G$ and $l$ are estimated for the colloidal deposits on soft and stiff substrates by using eq. 3 and eq. A1 (given in appendix) and tabulated in Table II. The estimated values of $G$ imply that the energy available for the propagation of cracks in particle deposits on the soft and stiff substrates are significantly different. In comparison, the energy release rate for the propagation of cracks on a stiff substrate is larger than that of the soft substrate. The origin of lower energy release rate on soft substrates can be attributed 

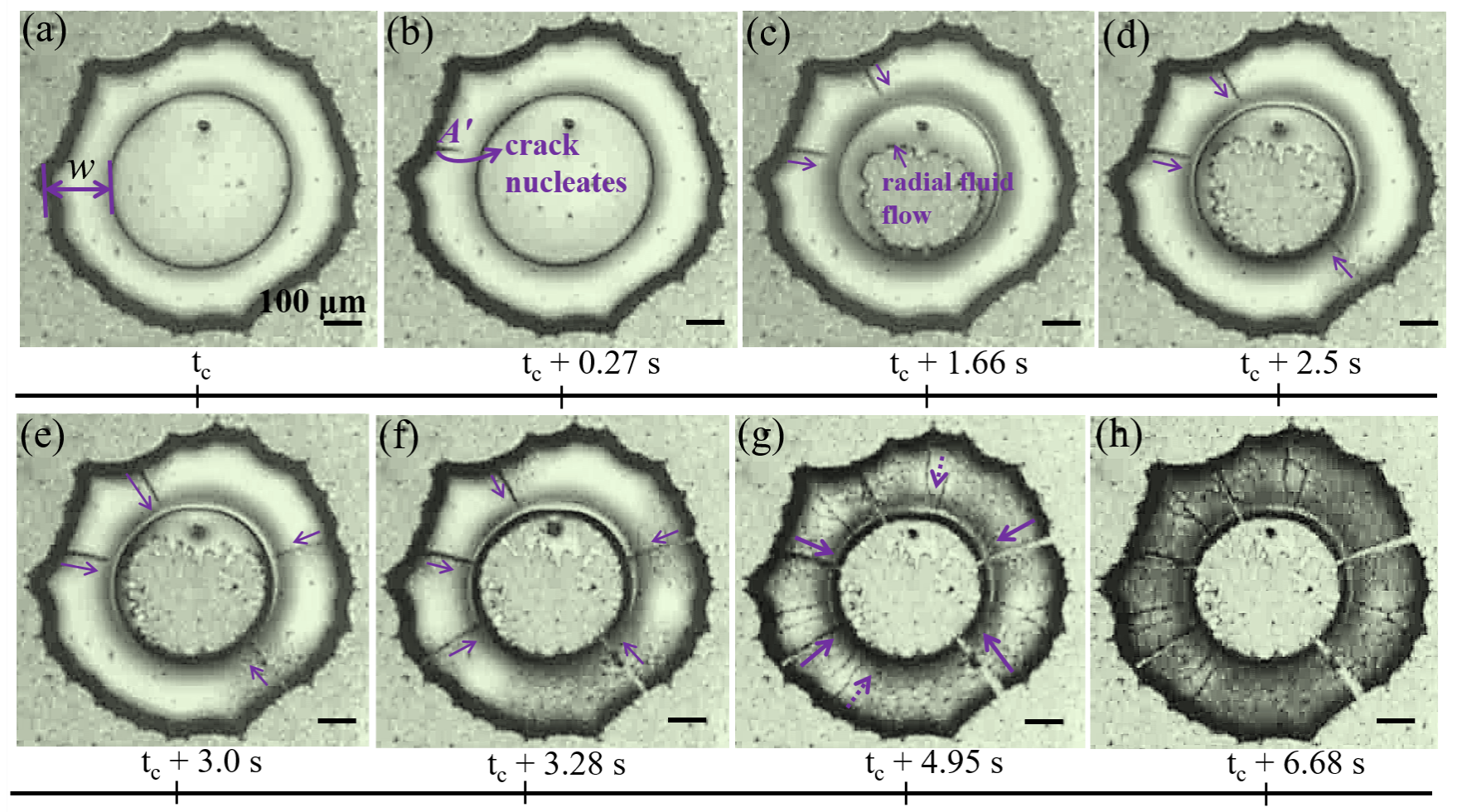

FIG. 6. (a) - (h) Snapshots show the propagation of cracks when colloidal silica dispersion's are dried on stiff substrates $\left(\mathrm{E}_{\mathrm{s}} \sim 70 \mathrm{GPa}\right)$. These Snapshots at different drying times are extracted from the a movie recorded by video-microscopy movie (see Supplementary Movie S2). The morphological evolution of the crack patterns formed in the particulate deposit at various instant of time starting from $t_{c}$ at which colloids solidifies into a gel-like can be seen. The width $w$ of the deposit and the nucleating point of cracks are marked. The direction of crack propagation is indicated by arrows.

TABLE II. The estimated value of reference length $l(\mu m)$ and the energy release rate $G\left(\mathrm{~J} / \mathrm{m}^{2}\right)$ is tabulated. The values of $G$ are close to reports of previous experiments on desiccation cracks [31].

\begin{tabular}{l||c|c|c|c|c}
\hline System & $\alpha$ & $\beta$ & $g(\alpha, \beta)$ & $l(\mu m)$ & $G\left(J / m^{2}\right)$ \\
\hline soft substrate & 0.99 & 0.24 & 23.85 & $37.41 \times H_{f}$ & 3.9 \\
\hline stiff substrate & -0.97 & -0.34 & 0.73 & $1.13 \times H_{f}$ & 6.7 \\
\hline
\end{tabular}

to its compliance nature. The particulate deposit on the soft substrate dissipates a part of the accumulated strain energy via the lateral contraction of the substrate surface, as evident from the formation of a crumpled region highlighted in Fig. 4(a) \& Fig. 4(b). In contrast, stiff substrates do not undergo any deformation, and almost all of the strain energy accumulated during the drying process is available for the propagation of cracks. Therefore, the different magnitude of $G$ entails that the mismatch in the modulus of elasticities between the colloidal deposit and the substrate affects the propagation of cracks, further manifesting as the variation in the crack morphology.

Now we turn to explain the contrasting crack patterns observed for the colloidal deposits on soft and stiff substrates, by invoking a rudimentary model taken from
Lame's problem $[32,33]$. As evident from the experiments, the cracks nucleate and propagate only after the formation of an annular gel-like semi-solid deposit, which possesses an axisymmetric cylindrical symmetry $(r, \theta, z)$. Therefore, we estimate stress and strain in the deposit of colloids by assuming it to be a coaxial cylinder with an inner radius being $r_{1}$, outer radius $r_{2}=r_{1}+w(w$ $=$ width of the annular region) as depicted in Fig. 7(a). Moreover, it also assumed that the particle deposits are isotropic and linear poroelastic in nature.

The equilibrium stresses in gel-like semi-solid state, prior to the nucleation of cracks are deduced from the following equations $[32,33]$ :

$$
\sigma_{\theta}=\sigma_{r}+r \frac{\partial \sigma_{r}}{\partial r} ; \quad \frac{\partial \sigma_{z}}{\partial z}=0
$$

where, $\sigma_{\theta}, \sigma_{r}$ and $\sigma_{z}$ are the hoop stress, the radial stress and the axial stress respectively. In principle, the dryinginduced stresses in the ring-shaped particulate deposits continuously evolve as the evaporation progresses. The stress modulation occurs due to the variation in local liquid pressure $\Pi(r, \theta, z, t)$ within the particulate deposit. This local liquid pressure reduces the interparticle distance and the colloids come closer until they come in physical contact. The local liquid pressure, $\Pi(r, \theta, z, t)$, exerted on the voids between the particles is expressed 
by a poroelastic equation [17, 34],

$$
\nabla^{2} \Pi=\frac{1}{D_{p}} \frac{\partial \Pi}{\partial t},
$$

where, $D_{p}$ is the consolidation coefficient. Now the condition of azimuthal symmetry (shown in Fig. 7(a)) and the slow temporal variation of pressure evident from the minute change in interparticle separation in the gel-like state gives rise to $\Pi(r, \theta, z, t) \equiv \Pi(r, z)$. As the height of the annular particulate deposit is much smaller than its radius, the variation of $\Pi(r, z)$ across the thickness of the deposit is negligible and hence $\Pi(r, z) \equiv \Pi(r)$. The axially averaged pressure across the thickness of the particulate deposit satisfies the boundary conditions: (i) $\left.\bar{\Pi}(r)\right|_{r=r_{2}}=P_{\circ}$, (ii) $\left.\frac{\partial \bar{\Pi}(r)}{\partial r}\right|_{r=r_{2}}=J_{s} \eta / k$, where, $P_{\circ}, J_{s}$, $\eta$ and $k$ are the atmospheric pressure, the evaporation rate, the solvent viscosity and the permeability of the particulate deposit respectively. Using these boundary conditions and solving eq. $5, \bar{\Pi}(r)$ is found to be,

$$
\bar{\Pi}(r)=\frac{r_{2} J_{s} \eta}{k} \ln \left(\frac{r}{r_{2}}\right)+P_{\circ} .
$$

Further, the stress, strain and the local liquid pressure generated in the particulate deposit are related by the Biot's constitutive relation [34, 35],

$$
\epsilon_{i j}=\frac{1}{E_{f}}\left[\left(1+\nu_{f}\right) \sigma_{i j}-\nu_{f} \sigma_{l l} \delta_{i j}\right]-\frac{\Pi}{3 K} \delta_{i j},
$$

where, $E_{f}$ is the Young's modulus of the particulate film, $\delta_{i j}$ is Kronecker delta, $K$ is bulk modulus of the colloidal deposit and the indices $\{i, j, l\}$ take the values from set $\{r, \theta, z\}$.

For the geometry considered here, the stresses in the particle deposit on the soft and stiff substrates (i) are in-plane i.e., $\sigma_{z}^{(s, h)} \rightarrow 0$, (ii) $\left.\sigma_{r}^{(s, h)}\right|_{r=r_{2}} \approx 0$, (iii) $\left.\sigma_{\theta}^{(s, h)}\right|_{r=r_{2}}=\left.\sigma_{r}^{(s, h)}\right|_{r=r_{2}}+\left.r \frac{\partial}{\partial r} \sigma_{r}^{(s, h)}\right|_{r=r_{2}}$. The superscript $s$ and $h$ in $\sigma^{(s, h)}$ denote the soft and stiff substrates respectively. We reiterate that lateral contraction of the substrate surface along with the colloidal deposit occur for sessile drops dried on soft substrates (shown in Fig. 7(a)). This allows us to make an assumption that the axial (along $z$ ) strain in the particulate deposit on soft substrate is less than the sum of lateral strains, i.e., $\epsilon_{z}^{(s)}<<\epsilon_{r}^{(s)}+\epsilon_{\theta}^{(s)}$. In contrast, for the film on a stiff substrate, the colloidal deposit is strongly pinned/adhered to the substrate (no-slip boundary condition) and the strain is solely arising from the evaporation driven shrinkage of the deposit, which happens predominately along the out of-plane direction (along z-axis). Therefore, the axial strain in the particulate film can be assumed to be greater than the sum of the lateral strains i.e. $\epsilon_{z}^{(h)}>>\epsilon_{r}^{(h)}+\epsilon_{\theta}^{(h)}$.

Finally, we estimate the in-plane stresses $\sigma_{r}$ and $\sigma_{\theta}$ for the particle deposit on soft and stiff substrates using the aforementioned boundary conditions. The inplane stresses are plotted as a function of the scaled radial distance $\mathrm{r} / \mathrm{r}_{2}$ and shown in Fig. 7(b) and Fig. 7(c).
A detailed derivation of the in-plane stresses and the various parameters used to estimate it is included in the appendix section. It is evident from Fig. 7(b) and Fig. 7(c) that as $r \rightarrow r_{2}$, both radial and hoop stresses tend to converge irrespective of the elasticity of the substrates. The tensile nature of the radial stress $\left(\sigma_{r}^{(s)}>0\right)$ and the compressive nature of the hoop stress $\left(\sigma_{\theta}^{(s)}\right)$ for particulate deposits on soft substrates are evident from Figure. 7(b). A contrasting behaviour is observed for particulate deposits on hard substrates, i.e., the radial stress is compressive $\left(\sigma_{r}^{(h)}<0\right)$ and hoop stress is tensile $\left(\sigma_{\theta}^{(h)}>0\right)$. Now we invoke the principle of fracture mechanics, which states that the "material cracks due to the accumulation of tensile stresses and the crack propagates in a direction perpendicular to the direction of the tensile stress" [36, 37]. Therefore, for the geometry of the particulate deposit employed here, the radial stress are tensile and hence will lead to circular cracks, while the tensile hoop stress will result in radial cracks. Fig. 7(b) and Fig. 7(c), obtained from analytical calculations of the in-plane stresses, clearly predict the formation of cracks along the azimuthal direction for colloidal deposits on soft substrates, and along the radial direction for the deposits on stiff substrates. The experimental observations of the crack patterns on the soft and stiff substrates are entirely in-line with the analytical predictions. Our combined experimental observations and the analytical calculations bring out the synergy between the substrate elasticity and the desiccation crack patterns.

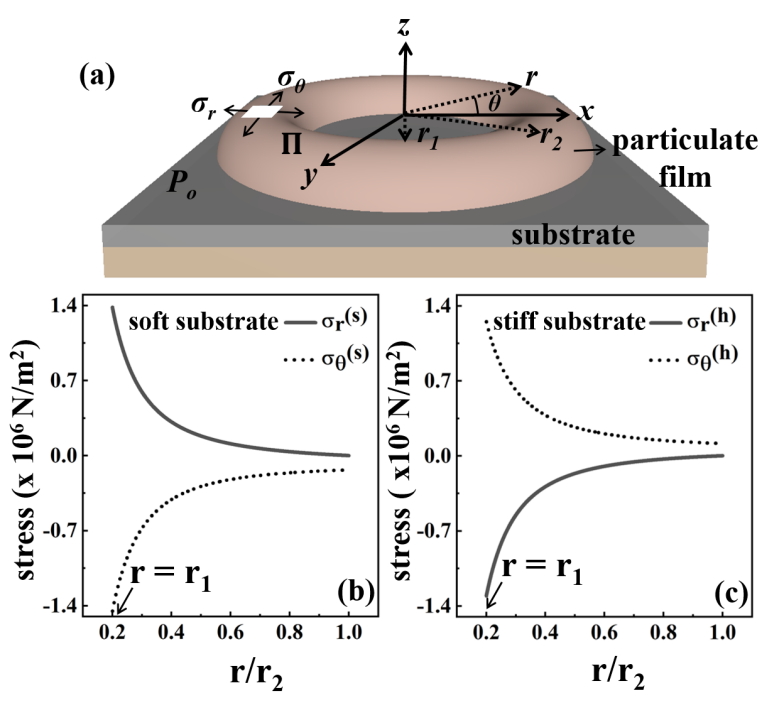

FIG. 7. (a) Three-dimensional schematic representation of the annular particulate deposit on soft or stiff substrates. The colloidal deposit is considered as a coaxial cylinder of inner radius $r_{1}$ and outer radius $r_{2}$. The direction of in-plane stresses $\sigma_{r}$ and $\sigma_{\theta}$ are marked. The in-plane stresses $\sigma_{r}, \sigma_{\theta}$ as a function of scaled radial distance $r / r_{2}$ developed in the particulate deposited on the soft substrate and on the stiff substrate are shown respectively in (b) and (c). The arrows point to the inner boundary of the annular colloidal deposit. 


\section{CONCLUSION}

In summary, our study reveals a remarkable change in the desiccation crack patterns in the colloidal deposits obtained by drying a pinned sessile drop containing colloids on elastomer (soft) and glass (stiff) substrates. Unique concentric circular cracks form in the colloids deposited on soft substrates, whereas linear cracks primarily oriented along the radial direction form for colloidal drops dried on stiff substrates. The dramatic change in the crack pattern, i.e., from radial-to-circular, solely arises from the variation of the underlying substrate's elasticity. The contrasting variation in desiccation crack patterns are explained by taking into account the nature of the drying induced in-plane stresses generated in the colloidal deposits. A full understanding of this intriguing phenomena requires further investigation of crack patterns by systematically tuning the softness of the substrates over a wide range, and on different verities of soft materials.

\section{ACKNOWLEDGEMENT}

H.L. acknowledges Indo-French Center for the Promotion of Advanced Research (IFCPAR) for the Raman Charpak Fellowship - 2018.

\section{APPENDIX}

\section{A. Dundur's parameter}

The Dundur's parameter $(\alpha, \beta)$ for an in-plane stress condition can be expressed as [16, 30],

$$
\begin{array}{r}
\alpha=\frac{E_{f}-E_{s}}{E_{f}+E_{s}}, \\
\beta=\frac{E_{f}\left(1-\nu_{s}\right)-E_{s}\left(1-\nu_{f}\right)}{E_{f}+E_{s}},
\end{array}
$$

where, $\mathrm{E}_{\mathrm{f}}$ and $\mathrm{E}_{\mathrm{s}}$ are the Young's modulus of the colloidal deposit and the substrate respectively, $\nu_{f}$ and $\nu_{s}$ are the Poisson's ratio of the colloidal deposit and substrate respectively. For our system, the Poisson's ratio; $\left.\nu_{s}\right|_{\text {stiff }} \approx 0.3,\left.\nu_{s}\right|_{\text {soft }} \approx 0.5$ and $\nu_{f} \approx 0.3[17]$.

\section{B. Calculation of in-plane radial and circumferential stress}

Nomenclature: $\sigma_{r}^{(s, h)}, \sigma_{\theta}^{(s, h)}, \sigma_{z}^{(s, h)}$ respectively are radial, circumferential and axial stresses in the deposit with the superscripts $s$ and $h$ respectively denoting the soft and stiff substrate.

$r_{1}, r_{2}$ respectively are the inner and the outer radii of the deposit $\epsilon_{r}^{(s, h)}, \epsilon_{\theta}^{(s, h)}, \epsilon_{z}^{(s, h)}$ respectively correspond to radial, circumferential and axial strains in the deposit. Once again, superscripts $s$ and $h$ are used to denote the soft and stiff substrate respectively.

$\Pi=$ Local pressure in the pores

$P_{\circ}=$ Atmospheric pressure

$P_{\text {cap }}=2 \gamma / R=$ Capillary pressure $\left(=10^{7} \mathrm{~N} / \mathrm{m}^{2}\right)$

$E_{f}=$ Young's modulus of the deposit

$\nu_{s}=$ Poisson's ratio of substrate

$\nu_{f}=$ Poisson's ratio of film

$K=$ Bulk modulus $\left[=E_{f} / 3(1-2 \nu)\right]$

$k=$ Permeability of the particulate deposit

$\eta=$ Solvent viscosity

$D_{p}=$ Consolidation coefficient or diffusivity $\left(=k E_{f} / \eta\right)$

$J_{s}=$ Evaporation rate

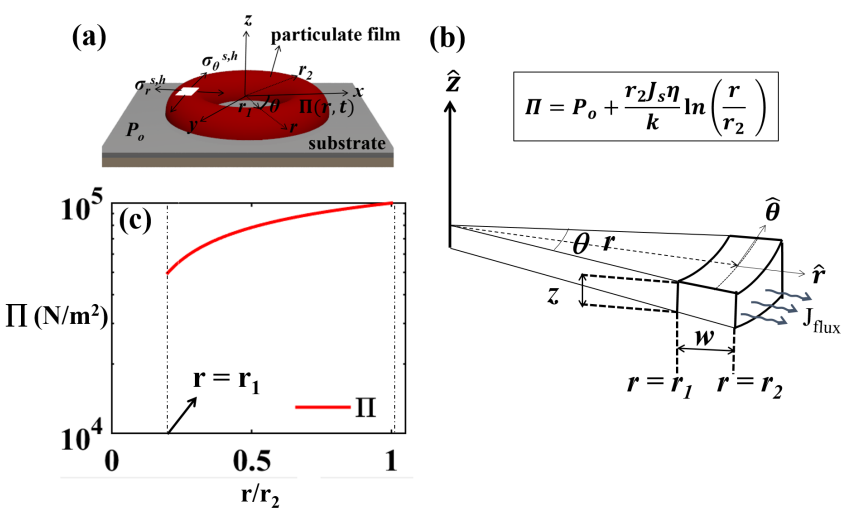

FIG. 8. (a) Schematic of an annular particulate deposit on a substrate. The atmospheric pressure is $\mathrm{P}_{\mathrm{o}}$ while the pressure in the pores of the particulate deposit is represented by $\Pi$. (b) A section of the annular particulate deposit displaying evaporation along radial direction is depicted. The expression for the pressure (П) inside the pores in the particulate deposit is also displayed. The pressure is estimated at an instant when the particulate deposit is semi-solid (gel like) and without cracks. The inner $\left(r_{1}\right)$ and outer $\left(r_{2}\right)$ radius of the particulate deposit, the width of the deposit $(w)$ and the evaporative flux $\left(J_{\text {flux }}\right)$ are marked. (c) The variation of pressure $\Pi$ with the scaled radial distance $\left(r / r_{2}\right)$ at a given instant is depicted. It shows that the pressure at $r=r_{2}$ is maximum and increases non-linearly as $r \rightarrow r_{2}$.

The particulate deposit is modeled as an isotropic linear poroelastic coaxial cylinder with an inner radius ' $r_{1}$ ', outer radius ' $r_{2}$ ' and thickness ' $H_{f}$ ' as shown in Fig. 8. The stresses and strains in the particulate deposit on soft/stiff substrates denoted respectively as $\left(\sigma_{r}^{(s, h)}, \sigma_{\theta}^{(s, h)}, \sigma_{z}^{(s, h)}\right)$ and $\left(\epsilon_{r}^{(s, h)}, \epsilon_{\theta}^{(s, h)}, \epsilon_{z}^{(s, h)}\right)$ are estimated when the film undergoes deformation in a gel-like semi-solid state prior to the nucleation of cracks. The equilibrium equation relating the stresses for the given 
geometry can be written as [32, 33],

$$
\begin{aligned}
\frac{\partial \sigma_{r}}{\partial r}+\frac{1}{r}\left(\sigma_{r}-\sigma_{\theta}\right) & =0 \\
\frac{\partial \sigma_{\theta}}{\partial r} & =0 \\
\frac{\partial \sigma_{z}}{\partial z} & =0
\end{aligned}
$$

In this model system, the particle assembly is assumed to experience the pressure $\Pi(r, \theta, z, t)$. The boundary conditions for the given system are [17],

$$
\left.\sigma_{r}\right|_{r=r_{2}} \approx P_{\circ}-\left.\Pi(r, \theta, z)\right|_{r=r_{2}}
$$

The local pressure in the pores of the particulate deposit, $\Pi$ at an instant when it is semi-dried, prior to the formation of cracks can be obtained from the poroelastic equation expressed as [17, 34],

$$
\nabla^{2} \Pi=0
$$

When a particle laden sessile drop dries, the fluid meniscus rapidly recedes inwards during the last stage of evaporation, which can be clearly observed in the supplementary video-S1. Therefore, the pressure in the particulate deposit can be assumed to vary only in the $r, \theta$ plane i.e. $\Pi(r, \theta, z) \equiv \Pi(r, \theta)$. Again, the particulate deposit has an azimuthal symmetry which results in $\Pi$ to be independent of $\theta$. This further allows us to approximate the differential equation, Eq A.6, as;

$$
\frac{1}{r} \frac{d}{d r}\left(r \frac{d \Pi}{d r}\right)=0
$$

which has a solution of the form:

$$
\Pi(r)=C_{1} \ln r+C_{2},
$$

where, $C_{1}$ and $C_{2}$ are integration constants, which can be obtained from the boundary conditions,

$$
\left.\Pi(r)\right|_{r=r_{2}} \approx P_{\circ} \text { and }\left.\frac{\mathrm{d} \Pi(\mathrm{r})}{\mathrm{dr}}\right|_{\mathrm{r}=\mathrm{r}_{2}}=\frac{\mathrm{J}_{\mathrm{s}} \eta}{\mathrm{k}} .
$$

The first boundary condition is based on assumption that the pressure at the edge of the particulate deposit is uniform and is equal to $P_{\circ}$. The second boundary condition directly comes from the Darcy's law. Using these boundary conditions, we obtain,

$$
\Pi(r)=\frac{r_{2} J_{s} \eta}{k} \ln \left(\frac{r}{r_{2}}\right)+P_{\circ} .
$$

The top layer of the particulate deposit is assumed to obey the slip boundary condition i.e. free to shrink and the layer adhered to the substrate obeys the noslip boundary condition i.e. constrained. The stresses and strains developed in the particulate deposit are expressed by the Biot's constitutive relations by assuming the system to be poroelastic and isotropic. The constitutive relation are as follows $[34,35]$ :

$$
\begin{aligned}
& \epsilon_{r}^{(s, h)}=\frac{1}{E_{f}}\left[\sigma_{r}^{(s, h)}-\nu_{f}\left(\sigma_{\theta}^{(s, h)}+\sigma_{z}^{(s, h)}\right)\right]-\frac{\Pi}{3 K} \\
& \epsilon_{\theta}^{(s, h)}=\frac{1}{E_{f}}\left[\sigma_{\theta}^{(s, h)}-\nu_{f}\left(\sigma_{r}^{(s, h)}+\sigma_{z}^{(s, h)}\right)\right]-\frac{\Pi}{3 K} \\
& \epsilon_{z}^{(s, h)}=\frac{1}{E_{f}}\left[\sigma_{z}^{(s, h)}-\nu_{f}\left(\sigma_{r}^{(s, h)}+\sigma_{\theta}^{(s, h)}\right)\right]-\frac{\Pi}{3 K}
\end{aligned}
$$

The sum of strains can be written as,

$\epsilon_{r}^{(s, h)}+\epsilon_{\theta}^{(s, h)}+\epsilon_{z}^{(s, h)}=\frac{1}{3 K}\left(\sigma_{r}^{(s, h)}+\sigma_{\theta}^{(s, h)}+\sigma_{z}^{(s, h)}\right)-\frac{\Pi}{K}$.

The annular particulate deposit is too thin to experience any noticeable effect of axial stress originating from the surrounding pressure. Therefore, we assume $\sigma_{z}^{(s, h)} \rightarrow 0$, and the planar stresses, i.e. $\left\{\sigma_{r}^{(s, h)}, \sigma_{\theta}^{(s, h)}\right\} \neq 0$. In the subsequent sections we derive the planar stresses for particulate deposits on the soft and stiff substrates respectively. All the quantities including the in-plane stresses and strains across the thickness are axially averaged.

Case-I: In-plane stresses for particulate deposits on soft substrate The sum of in-plane strains generated in the particulate deposit is assumed to be greater than the axial strain, i.e. $\epsilon_{z}^{(s)}<<\epsilon_{r}^{(s)}+\epsilon_{\theta}^{(s)}$. This approximation is valid for the present scenario as the film together with the substrate can undergo lateral shrinkage causing the substrate to undergo deformation. From the constitutive relations and using Eq. A.14, we obtain,

$$
\epsilon_{r}^{(s)}+\epsilon_{\theta}^{(s)}=\frac{1}{3 K}\left[\sigma_{r}^{(s)}+\sigma_{\theta}^{(s)}\right]-\frac{\Pi}{K} .
$$

Substituting Eq. A.11 and Eq. A.12 in Eq. A.15:

$$
\Longrightarrow \sigma_{r}^{(s)}+\sigma_{\theta}^{(s)}=-\frac{\Pi A_{1}}{3 K}
$$

where, $A_{1}=\left(\frac{1}{E_{f}}-\frac{\nu_{f}}{E_{f}}-\frac{1}{3 K}\right)^{-1}$.

Substituting Eq. A.2 and Eq. A.10 in Eq. A.16 we obtain,

$$
\frac{d}{d r}\left(r^{2} \sigma_{r}^{(s)}\right)=-r B_{1} \ln \frac{r}{r_{2}}-r P_{\circ} \frac{A_{1}}{3 K} .
$$

where, $B_{1}=\frac{A_{1} r_{2} J_{s} \eta}{3 K k}$.

Integrating Eq. A.17, substituting integration constant using Eq. A.5 and Eq. A.10, we find,

$$
\sigma_{r}^{(s)}=-B_{1}\left\{-\frac{1}{4}+\frac{1}{4} \frac{r_{2}^{2}}{r^{2}}+\frac{1}{2} \ln \frac{r}{r_{2}}\right\}-\frac{P_{\circ} A_{1}}{6 K}\left(1-\frac{r_{2}^{2}}{r^{2}}\right) .
$$

Now substituting $\sigma_{r}^{(s)}$ in Eq. A.2, we obtain $\sigma_{\theta}^{(s)}$ :

$$
\sigma_{\theta}^{(s)}=\sigma_{r}^{(s)}+B_{1}\left\{-\frac{1}{2}+\frac{r_{2}^{2}}{2 r^{2}}\right\}-\frac{P_{\circ} A_{1}}{3 K} \frac{r_{2}^{2}}{r^{2}}
$$


Case- II: In plane stresses for particulate deposits on stiff substrates As mentioned earlier, the stiff substrates are not deformable. The particulate deposit is strongly pinned/adhered to the substrate (noslip boundary condition) and the strain is solely arising from the evaporation driven shrinkage of the layer, which happens predominately along the out of-plane direction (along the z-axis shown in Fig. 8). Therefore, for colloid deposits on stiff substrates, we assume that the sum of the lateral strains is much smaller than the axial strain i.e. $\epsilon_{z}^{(h)}>>\epsilon_{r}^{(h)}+\epsilon_{\theta}^{(h)}$. Now the constitutive relation appropriate for the given condition can be obtained by substituting $\sigma_{z}=0$ in Eq. A.13,

$$
\epsilon_{z}^{(h)}=\frac{1}{E_{f}}\left[-\nu_{f}\left(\sigma_{r}^{(h)}+\sigma_{\theta}^{(h)}\right)\right]-\frac{\Pi}{3 K},
$$

and from Eq. A.14,

$$
\epsilon_{z}^{(h)}=\frac{1}{3 K}\left[\sigma_{r}^{(h)}+\sigma_{\theta}^{(h)}\right]-\frac{\Pi}{K}
$$

Equating Eq. A.20 and Eq. A.21 we obtain,

$$
\sigma_{r}^{(h)}+\sigma_{\theta}^{(h)}=A_{2} \Pi
$$

where, $A_{2}=\frac{2}{3 K\left(\frac{1}{3 K}+\frac{\nu_{f}}{E_{f}}\right)}$

Now by substituting Eq. A.2 and Eq. A.10 in Eq. A.22,

$$
\frac{d}{d r}\left(r^{2} \sigma_{r}^{(h)}\right)=\left[B_{2} r \ln \frac{r}{r_{2}}+A_{2} r P_{\circ}\right]
$$

$B_{2}=\frac{A_{2} r_{2} J_{s} \eta}{k}$

Integrating both side and substituting the value of integration constants, obtained from Eq. A.5 and Eq. A.10,

$$
\sigma_{r}^{(h)}=B_{2}\left\{\frac{1}{2} \ln \frac{r}{r_{2}}-\frac{1}{4}+\frac{r_{2}^{2}}{4 r^{2}}\right\}+\frac{A_{2} P_{\circ}}{2}\left\{1-\frac{r_{2}^{2}}{r^{2}}\right\}
$$

Now substituting $\sigma_{r}^{(h)}$ in the Eq. A.2 gives the circumferential/hoop stress;

$$
\sigma_{\theta}^{(h)}=\sigma_{r}^{(h)}+B_{2}\left[\frac{1}{2}-\frac{r_{2}^{2}}{2 r^{2}}\right]+\frac{A_{2} P_{\circ} r_{2}^{2}}{r^{2}}
$$

The in-plane stresses are obtained by substituting $J_{s} \sim 10^{-8} \mathrm{~m} / \mathrm{s}$ [17], $r_{1}, r_{2}$ as obtained from the microscopy image and the $P_{\circ} \sim 10^{5} \mathrm{~N} / \mathrm{m}^{2}, \eta \sim 10^{-3} \mathrm{Pa.s}$, $E_{f} \sim 1 \mathrm{GPa}, \nu_{f} \sim 0.3, k \sim 10^{-18} \mathrm{~m}^{2}[17]$.
[1] W. Narr and J. Suppe, J. Struct. Geo. 13, 1037 (1991).

[2] T. Bai, D. Pollard, and H. Gao, Nature 403, 753 (2000).

[3] A. Groisman and E. Kaplan, Eur. Phys. Lett. 25, 415 (1994).

[4] S. Bohn, L. Pauchard, and Y. Couder, Phys. Rev. E 71, 046214 (2005).

[5] G. Müller, J. Volcano. and Geo. Res. 86, 93 (1998).

[6] L. Goehring, L. Mahadevan, and S. W. Morris, Proc. Nat. Acad. Sci. 106, 387 (2009).

[7] C. Allain and L. Limat, Phys. Rev. Lett. 74, 2981 (1995).

[8] L. Goehring, W. J. Clegg, and A. F. Routh, Soft Matter 7, 7984 (2011).

[9] K.-T. Leung, L. Jozsa, M. Ravasz, and Z. Neda, Nature 410, 166 (2001).

[10] L. Goehring, A. Nakahara, T. Dutta, S. Tarafdar, and S. Kitsunezaki, Desiccation cracks and their patterns: Formation and Modelling in Science and Nature (John Wiley \& Sons, 2015).

[11] J. Zarzycki, J. Non Cryst. Sol. 100, 359 (1988).

[12] M. S. Tirumkudulu and W. B. Russel, Langmuir 21, 4938 (2005).

[13] W. P. Lee and A. F. Routh, Langmuir 20, 9885 (2004).

[14] K. B. Singh and M. S. Tirumkudulu, Phys. Rev. Lett. 98, 218302 (2007).

[15] T. L. Anderson, Fracture Mechanics: Fundamentals and Applications, Third Edition (Taylor \& Francis, New York, NY, 2005).

[16] J. Beuth Jr, Int. J. Sol. Struc. 29, 1657 (1992).

[17] F. Giorgiutti-Dauphiné and L. Pauchard, Eur. Phys. J. E 37 (2014).
[18] M. Smith and J. Sharp, Langmuir 27, 8009 (2011).

[19] G. Jing and J. Ma, J. Phys. Chem. B 116, 6225 (2012).

[20] S. L. Sanjay, B. G. Annaso, S. M. Chavan, and S. V. Rajiv, Journal of Surface Engineered Materials and Advanced Technology 2012 (2012).

[21] R. D. Deegan, O. Bakajin, T. F. Dupont, G. Huber, S. R. Nagel, and T. A. Witten, Nature 389, 827 (1997).

[22] R. D. Deegan, O. Bakajin, T. F. Dupont, G. Huber, S. R. Nagel, and T. A. Witten, Phys. Rev. E 62, 756 (2000).

[23] H. Lama, V. R. Dugyala, M. G. Basavaraj, and D. K. Satapathy, Phys. Rev. E 94, 012618 (2016).

[24] H. Lama, M. G. Basavaraj, and D. K. Satapathy, Soft Matter 13, 5445 (2017).

[25] P. t. Bacchin, Eur. Phys. J. E 41, 94 (2018).

[26] W. C. Oliver and G. M. Pharr, J. Mater. Res. 7, 1564 (1992).

[27] A. C. Fischer-Cripps, Time-dependent Nanoindentation (Springer, 2011).

[28] See Supplemental Material at http:// for supplementary movies.

[29] Z. C. Xia and J. W. Hutchinson, J. Mech. Phys. Sol. 48, 1107 (2000).

[30] S. Schmauder and M. Meyer, Z. Metallkd 83, 525 (1992).

[31] L. Goehring, W. J. Clegg, and A. F. Routh, Phys. Rev. Lett. 110, 024301 (2013).

[32] S. Timoshenko and J. Goodier, Theory of Elasticity (McGraw-Hill, 1951).

[33] M. Tabesh, B. Liu, J. Boyd, and D. Lagoudas, Smart Mater. Struc. 22, 094007 (2013).

[34] M. A. Biot, J. Appl. Phys. 12, 155 (1941). 
[35] G. W. Scherer, J. Non Crystal. Sol. 109, 171 (1989), ISSN 0022-3093.

[36] J. W. Hutchinson and Z. Suo, Adv. Appl. Mech. 29, 63 (1991).
[37] J. Marthelot, B. Roman, J. Bico, J. Teisseire, D. Dalmas, and F. Melo, Phys. Rev. Lett. 113, 085502 (2014). 